\title{
Adaptive supply management with constraints
}

\author{
Galina N.Reshetnikova \\ National Research Tomsk State University \\ Tomsk, Russia
}

Nadezhda Yu. Khabibulina

National Research Tomsk Polytechnic University

Tomsk, Russia

e-mailhnu@tpu.ru

\author{
Vladislav P. Kotcubinskiy \\ National Research Tomsk Polytechnic University \\ Tomsk, Russia
}

\begin{abstract}
Construction of a mathematical model is carried out and the adaptive tracking supply control system is considered on the basis of a local quadratic criterion. A system of restrictions is proposed that allows to avoid both excess and insufficient volume of goods in the warehouse. The results of numerical simulation are presented.
\end{abstract}

Key words - mathematical model, supply management, local criteria, constraints

\section{INTRODUCTION}

The use of informational technology can improve the efficiency of any economic system, primarily through the ability to collect, process, store and analyze information, as well as make managerial decisions. There is an increasing need to use automatic control systems for a well-timed reaction to changes in the market situation, while control algorithms developed for technical systems can be successfully used for managing economic processes. The main difficulty lies in the construction of adequate mathematical models.

There have been significant changes in the sphere of commodity circulation due to the fact that new methods and technologies for the supply of goods that are based on the concept of logistics have begun to be used in recent years [1]. Logistics supply is one of the main logistics subsystems and studies the process of the movement of raw materials, materials, components from the procurement market to the warehouses of the enterprise. At the same time, it is necessary to forecast the demand and form the logistics schemes in such a way as to reduce the physical and intermediary costs.

At present, dynamic marketing tasks are becoming increasingly important, in which economic processes are described by differential or difference equations. This allows us to use the theory of control in the state space to solve many problems with computers use. In particular servo element systems are used for the synthesis of control actions based on the method of analytical design of optimal controllers when optimizing the mathematical expectation of various functionals. At the same time, due to the absence of a piece of information, it becomes necessary to use estimates of the state and model parameters.

Various methods for solving supply control problems are discussed in [2-6]. In this paper, we propose using supplycontrol algorithms based on a local quadratic criterion in order to maintain the desired volume of goods in the trading company's warehouse.

\section{MATHEMATICAL MODEL CONSTRUCTION}

We propose a mathematical model for supply management in a trading company that purchases, stores and sells goods.

Let $U(t)$ - the volume of the delivered goods in purchase prices per unit of time, $z(t), v(t), Y(t)$ - the volumes of the goods in sales prices at the warehouse of the trading company, the consumer and the potential demand for the supplied goods. Equations for the dynamics of goods volume in the warehouse, the consumer and income can be written in the form [7]:

$$
\begin{aligned}
& z(t)=k_{3} U(t)-k_{4}(Y(t)-v(t)) z(t)-k_{1} z(t), z\left(t_{0}\right)=z_{0} \\
& \&(t)=k_{4}(Y(t)-v(t)) z(t)-k_{2} v(t), v\left(t_{0}\right)=v_{0} \\
& \&(t)=k_{4}(Y(t)-v(t)) z(t)-U(t)-k_{5} z(t), w\left(t_{0}\right)=w_{0},
\end{aligned}
$$

where $k_{1}, k_{2}, k_{3}, k_{4}, k_{5}$ - coefficients that characterize the spoilage of goods during storage; consumption rate; trade margins; rate of sales; charge for goods storage. The income consists of the difference from the volume of the goods that was sold at the selling price, delivered at the purchase price and storage costs. Equation $k_{4}(Y(t)-v(t)) z(t)$ sets the volume of the goods that was sold per time unit.

Let's write equation (1) in the form of a system of ordinary differential stochastic equations of the form:

$$
\mathscr{L}(t)=\bar{A}(t) x(t)+\bar{B}(t) u(t)+\bar{F}(t) q(t), x\left(t_{0}\right)=x_{0}
$$

where the state vector $x(t)=(z(t), v(t), w(t))^{\mathrm{T}} \quad$ characterizes the volume of goods in the warehouse, the consumer and income; control $u(t)=U(t)$ specifies the scope of supply. Linearization of the system (1) is carried out by Taylor method with relating to the functions $\varphi_{z}(t), \varphi_{v}(t), \varphi_{y}(t)$, which describe the estimated values of the goods in the warehouse, the consumer and potential demand. While the matrices of the dynamic properties $\bar{A}(t)$ and influence of control actions $\bar{B}(t)$ in (2) have the following form: 


$$
\bar{A}(t)=\left(\begin{array}{ccc}
-k_{4}\left(\varphi_{y}(t)-\varphi_{v}(t)\right)-k_{1} & k_{4} \varphi_{z}(t) & 0 \\
k_{4}\left(\varphi_{y}(t)-\varphi_{v}(t)\right) & -k_{4} \varphi_{z}(t)-k_{2} & 0 \\
k_{4}\left(\varphi_{y}(t)-\varphi_{v}(t)\right)-k_{5} & -k_{4} \varphi_{z}(t) & 0
\end{array}\right), \quad \bar{B}(t)=\left(\begin{array}{c}
k_{3} \\
0 \\
-1
\end{array}\right)
$$

Vector $q(t) \in R^{h_{1}}$ describes the effect of random factors that we will specify by a vector of Gaussian random variables with characteristics:

$$
\begin{aligned}
& M\{q(t)\}=\bar{q}(t), \\
& M\left\{(q(t)-\bar{q}(t))(q(\tau)-\bar{q}(\tau))^{\mathrm{T}}\right\}=Q(t) \delta(t-\tau),
\end{aligned}
$$

matrix $\bar{F}(t)$, determining the influence of random factors in the model, is specified specifically for the problem that is being solved, $\delta(t-\tau)$ - delta-function of Dirac.

For supply management with use of information technology, for (2) a discrete model is built

$$
x(k+1)=A(k) x(k)+B(k) u(k)+F(k) q(k), x(0)=x_{0},
$$

where $A(k)=I_{3}+\Delta t \bar{A}\left(t_{k}\right), B(k)=\Delta t \bar{B}\left(t_{k}\right), F(k)=\sqrt{\Delta t} \bar{F}\left(t_{k}\right)$, $\Delta t=t_{k+1}-t_{k}-$ sampling interval, $I_{3}-$ unit matrix of the third order, $q(k)$ - a sequence of Gaussian random variables with characteristics:

$$
\begin{aligned}
& M\{q(k)\}=\bar{q}(k), \\
& M\left\{(q(k)-\bar{q}(k))(q(j)-\bar{q}(j))^{\mathrm{T}}\right\}=Q(k) \delta_{k, j},
\end{aligned}
$$

where $k$ corresponds to the time moment $t_{k}=t_{0}+k \Delta t, k=\overline{0, N}, \delta_{k, j}-$ Kronecker's symbol.

\section{MANAGEMENT AlgorithMS}

Supply management in real conditions is carried out using information that is often incomplete and contains errors. Let's assume that the mathematical model of the information system has the form:

$$
y(k)=H x(k)+r(k)
$$

where $y(k) \in R^{l},(l \leq 3)$ - vector, that has some information, $H \in R^{l \times 3}$ - matrix, it consists of zeros and ones whose zero columns indicate the lack of information, $r(k) \in R^{l}$ - vector, defining the errors of the information system, which are described by a sequence of Gaussian random variables with characteristics:

$$
M\{r(k)\}=0, M\left\{r(k) r^{T}(j)\right\}=R \delta_{k j} .
$$

In addition, it is necessary to take into account the presence in the model of variables in time of unknown parameters that form a vector $\theta(t) \in R^{N_{R}}$. In this regard, for the formation of control actions, let's construct estimates of the state vectors and parameters $\hat{x}(k), \hat{\theta}(k)$ at discrete instants of time from the current information $y(k)$. It is assumed here that the a priori distributions of the vectors of the initial conditions $x_{0}$ and $\theta(0)$ are Gaussian:

$$
\begin{gathered}
M\left\{x_{0}\right\}=\bar{x}_{0}, M\left\{\left(x_{0}-\bar{x}_{0}\right)\left(x_{0}-\bar{x}_{0}\right)^{T}\right\}=P_{\mathrm{x}}, \\
M\{\theta(0)\}=\bar{\theta}(0), M\left\{(\theta(0)-\bar{\theta}(0))(\theta(0)-\bar{\theta}(0))^{T}\right\}=P_{\theta},
\end{gathered}
$$

where $P_{\mathrm{x}}, P_{\theta}-$ covariance matrices of errors of initial conditions. To construct the estimates, let's use Kalman's discrete parallel filters, the algorithms of which are given in [8].

The recurrent algorithm for state estimate has the following form:

$$
\begin{aligned}
& \hat{x}(k+1)=\hat{x}(k+1 / k)+K(k)[y(k+1)-H \hat{x}(k+1 / k)], \\
& \hat{x}(k+1 / k)=A(k, \hat{\theta}(k)) \hat{x}(k)+B(k, \hat{\theta}(k)) u(k)+F(k) \bar{q}(k), \hat{x}(0)=\bar{x}_{0}, \\
& K(k)=P_{x}(k+1 / k) H^{T}\left[H P_{x}(k+1 / k) H^{T}+R\right]^{-1}, \\
& P_{x}(k+1 / k)=A(k, \hat{\theta}(k)) P_{x}(k) A^{T}(k, \hat{\theta}(k))+F(k) Q(k) F^{T}(k), \\
& P_{x}(k+1)=\left[I_{3}-K(k) H\right] P_{x}(k+1 / k), \\
& P_{x}(0)=P_{x_{0}} .
\end{aligned}
$$

In (8) $\hat{x}(k+1 / k)$ - extrapolated estimate of state vector $x(k+1), \quad P_{x}(k+1 / k)$ - prediction of dispersion $P_{x}(k)$ one step forward, $K(k)$ - matrix of the filter gain.

The recurrent algorithm for parameters estimation of the model has the following form

$$
\begin{aligned}
& \hat{\theta}(k+1)=\hat{\theta}(k)+L(k)[y(k+1)-H \Phi(\hat{x}(k), u(k)) \hat{\theta}(k)- \\
& -H f(\hat{x}(k), u(k))], \quad \hat{\theta}(0)=\bar{\theta}_{0}, \\
& L(k)=P_{\theta}(k) \Phi^{T}(\hat{x}(k), u(k)) M^{-1}, \\
& M(k)=H \Phi(\hat{x}(k), u(k)) P_{\theta}(k) \Phi^{T}(\hat{x}(k), u(k)) H^{T}+ \\
& +H F(k) Q(k) F^{T}(k) H^{T}+R, \\
& P_{\theta}(k+1)=\left[I_{N_{k}}-L(k) H \Phi(\hat{x}(k), u(k))\right] P_{\theta}(k), \\
& P_{\theta}(0)=P_{\theta_{0}} \text {. }
\end{aligned}
$$

In (9) $L(k)$ - matrix of filter amplification, matrix $\Phi(\cdot) \in R^{3 \times N_{R}}$ and vector $f(\cdot) \in R^{3}$ are obtained by presenting the system (5) in the form:

$$
\begin{aligned}
& x(k+1)=\Phi(x(k), u(k)) \theta(k)+f(x(k), u(k))+F(k) q(k), \\
& x(0)=x_{0}
\end{aligned}
$$

To form the scope of supply, let's use a servo element system that allows us to track a part of the components of the 
vector of a given state. Such problems for technical systems were considered in $[9,10,11]$.

Let matrix $S \in R^{p \times 3}$ rank $p(p<3)$ consists of zeros and ones whose zero columns indicate those components of the state vector that are not tracked in the synthesis of control actions. Supply management will be implemented on the basis of minimizing the mathematical expectation of a local quadratic criterion

$$
I^{(S)}(k)=M\left\{\left(S x(k+1)-x_{z}^{(S)}(k)\right)^{T} C^{(S)}\left(S x(k+1)-x_{z}^{(S)}(k)\right)+u^{T}(k) D u(k)\right\},
$$

where $C^{(S)} \in R^{p \times p}$ nonnegative definite weight matrix, $D>0$ weight coefficient, $x_{z}^{(S)}(k) \in R^{p}$ tracking vector.

Then the management that determines the volume of supply is defined as follows

$$
\begin{aligned}
u(k)=-\left(B^{T}(k)\right. & \left.S^{T} C^{(S)} S B(k)+D\right)^{-1} B^{T}(k) S^{T} C^{(S)} \times \\
& \times\left(S A(k) x(k)+S F(k) \bar{q}(k)-x_{z}^{(S)}(k)\right) .
\end{aligned}
$$

\section{FORMATION OF RESTRICTIONS}

The presence of constraints is a characteristic feature of economic systems, while there are the constraints for the state vector and control.

Based on the economic sense, for the first two components of the state vector, characterizing the volume of goods in the warehouse and the consumer, are constrained by the form:

$$
\tilde{x}_{i}(k)=\left\{\begin{array}{l}
x_{i}(k), x_{i}(k) \geq 0, \\
0, x_{i}(k)<0, \quad i=\overline{1,2} .
\end{array}\right.
$$

Deliveries will be formed when tracking the desired volume of goods in the warehouse and in such a way as to avoid both the excess and the deficit of the goods in the warehouse, which leads to losses. In the first case, damage to the goods may occur, and in the second case, losses may arise due to the lack of goods in the presence of demand. In this case $p=1, S=\left(\begin{array}{lll}1 & 0 & 0\end{array}\right), x_{z}^{(S)}(k)=z_{z}(k)$-desired volume of goods in stock [8].

Let's assume that the goods are delivered only in whole packages, the delivery time is constant, equal to $j \Delta t$ and the payment for the goods is carried out in fact, i.e. at the time of receipt of the goods.

Define the estimated rate of sales and an estimate of the volume of goods in the warehouse without a new delivery as follows:

$$
\begin{aligned}
& p_{R}(k)=k_{4}\left(\varphi_{y}(k)-\varphi_{v}(k)\right) z_{z}(k), \\
& \hat{g}(k)=\hat{z}(k)-j p_{R}(k)-\delta
\end{aligned}
$$

where $\delta$ - the size of the insurance stock, which determines the volume of goods to account for random factors.

The estimated number of days and an estimate of the number of days of work on commodity balances are given in the form:

$$
T_{R}(k)=\left[\frac{z_{z}(k)+\delta j}{p_{R}(k)}\right], \quad \hat{T}(k)=\left[\frac{\hat{z}(k)}{p_{R}(k)}\right] .
$$

Then if $T_{R}(k)<\hat{T}(k)$, then the goods are not supplied, i.e. it is assumed $u(k)=0$. If $T_{R}(k) \geq \hat{T}(k)$, then there is a delivery of goods. In this case, it is formed according to (12) preliminary delivery $u(k)$, which is then specified.

Let $z_{\max }(k), z_{\min }(k)$ - the values specifying the maximum and minimum volume of the goods that can be stored in the warehouse. These values are determined by the entrepreneur, based on the area of the warehouse, the availability of funds and the situation on the market.

The scope of supply is specified with the consistent use of two inequalities:

$$
u^{(1)}(k)=\left\{\begin{array}{l}
u(k), \hat{g}(k)+u(k) \leq z_{\text {max }}(k), \\
z_{\text {max }}(k)-\hat{g}(k)-u(k), \hat{g}(k)+u(k)>z_{\text {max }}(k),
\end{array}\right.
$$

and

$$
u^{(2)}(k)=\left\{\begin{array}{l}
0, u^{(1)}(k)<z_{\text {min }}(k), \\
u^{(1)}(k), z_{\text {min }}(k) \leq u^{(1)}(k) \leq z_{\text {max }}(k), \\
z_{\text {max }}(k), u^{(1)}(k)>z_{\text {max }}(k) .
\end{array}\right.
$$

Because the goods are delivered in whole packages, this must be taken into account when determining the scope of supply. Let $s$-packing cost of goods. Then the expressions are as following

$$
\mu(k)=\left[\frac{u^{(2)}(k)}{s}\right], s^{*}(k)=u^{(2)}(k)-\mu(k) s
$$

determine the number of whole packages in the delivery and the remainder.

The scope of supply in whole packages will be determined as follows:

$$
u^{(3)}(k)=\left\{\begin{array}{l}
0, \mu(k)=0, s^{*}(k) \leq 0,5 s \\
\mu(k) s, \mu(k) \neq 0, s^{*}(k) \leq 0,5 s \\
(\mu(k)+1) s, \mu(k) \neq 0, s^{*}(k)>0,5 s
\end{array}\right.
$$

The final delivery volume is determined by taking into account the carrying capacity of the vehicles carrying the goods and the economic feasibility of the delivery: 


$$
u^{(4)}(k)=\left\{\begin{array}{l}
0, u^{(3)}(k)<U_{\text {min }}, \\
u^{(3)}(k), U_{\min } \leq u^{(3)}(k) \leq U_{\text {max }}, \\
U_{\text {max }}, u^{(3)}(k)>U_{\text {max }} ;
\end{array}\right.
$$

where $U_{\max }$ - indicates the maximum delivery, but $U_{\min }-$ on the minimum economically justified scope of supply.

\section{RESULTS OF NUMERICAL SIMULATION}

For numerical simulation the work of a trading company that specializes in the supply and sale of auto parts were used. What is more, the review included a time period, when the preparation of cars for the spring-summer season was carried out, i.e. the need for spare parts increased. In this connection, the values $z_{\text {max }}(k), z_{\text {min }}(k), z_{z}(k)$, which determine the maximum, minimum and desired quantities of goods in the warehouse of the trading company, are given functions that depend on the time. This indicates that the trading company in the period of increased demand increases the amount of storage space allocated for the storage of a particular product. In addition, only the information on the volume of goods in the warehouse was used for the formation of supplies and unknown parameters in the model were coefficients that set the pace of sales and consumption.

Fig. 1 shows the graphs that define $z_{\max }(k), z_{\min }(k), z_{z}(k)-$ the maximum, minimum and desired quantities of goods in the warehouse at each moment $k$ and the dynamics of the volume of goods in the warehouse of the trading company, as well as the volumes of supplies.

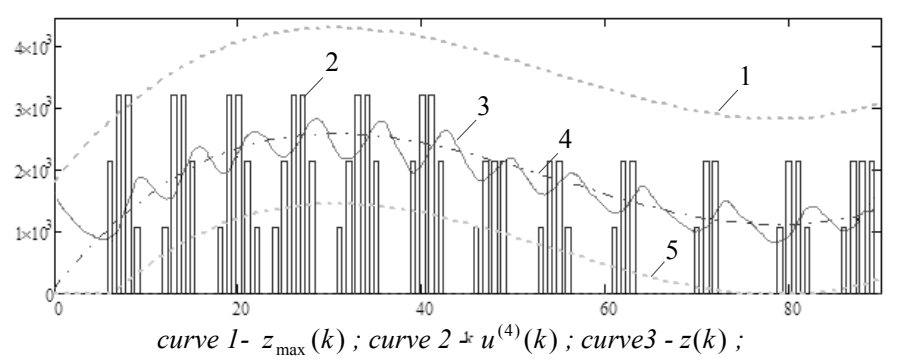

$$
\text { curve } 4-z_{z}(k) \text {; curve } 5-z_{\min }(k)
$$

Fig. 1. The simulation results

The above results illustrate the operability and quality of the proposed algorithms.

\section{CONCLUSION}

In this paper, a mathematical model for supply management using the methods of automatic control theory was proposed. The volume of deliveries is determined on the basis of the modification of the local quadratic criterion while tracking part of the components of the state vector. A stochastic information system that allows incomplete information, and the presence of unknown parameters of the model, necessitate the use of state estimates and parameters for the construction of which Kalman filters are used.

Supply management is carried out in order to keep the desired amount of goods in the warehouse of the trading company, thus it is necessary to avoid both surplus and deficit of goods in the warehouse, which leads to losses. To achieve this, a system of time and volume constraints is proposed.

The results of modeling using the data of the work of a particular company illustrate the operability and quality of the proposed model and control algorithms.

\section{REFERENCES}

[1] Lukinsky, V.S. Models and methods of the theory of logistics. St. Petersburg, Piter, 2008. 448 p.

[2] Smagin, V.I., Koshkin, G.M., Kim, K.S. Locally Optimal Inventory Control with Time Delay in Deliveries and Incomplete Information on Demand. Proc. Second International Symposium on Stochastic Models in Reliability Engineering, Life Science and Operations Management. Beer Sheva, Israel. 2016, pp. 570-574.

[3] Aggelogiannaki, E., Doganis, Ph., Sarimveis, H. An adaptive model predictive control conguration for production-inventory systems. Int. J. of Production Economics, 2008, vol. 114, pp. 165-178.

[4] Henneta, J.-C. A globally optimal local inventory control policy for multistage supply chains. Int. J. of Production Research, 2009, vol. 47, issue 2, pp. 435-453.

[5] Um, J. Improving supply chain flexibility and agility through variety management. The International Journal of Logistics Management, 28(2), pp.464-487. Available at: http://dx.doi.org/10.1108/ijlm-07-2015-0113 (accessed September 2, 2017)

[6] Babaei, A., Shahanaghi, K. A new model for planning the distributed facilities locations under emergency conditions and uncertainty space in relief logistics. Uncertain Supply Chain Management, pp.105-125. Available at: http://dx.doi.org/10.5267/j.uscm.2016.10.004 (accessed August 15, 2017)

[7] Reshetnikova, G.N. Adaptive control of delivery and advertising. Algorithms. Models. Limitations. Monograph. Saarbrucken (Germany), LAP LAMBERT Academic Publishing, 2012,152 p.

[8] Brammer, H., Siffling G. The Kalman-Busch filter. Moscow: Nauka Publ., 1972, 200 p.

[9] Reshetnikova, G. N., Khabibulina, N. Yu., Kotcubinskiy, V. P., Polonskaya, M. S. Servo-System simulation. Proc. of the International Conference Mechanical Engineering, Automation and Control Systems (MEACS). Tomsk, National Research Tomsk Polytechnic University; Institute of Electrical and Electronics Engineers (IEEE). IEEE, 2015, 4 p. Available at: http://dx.doi.org/10.1109/MEACS.2015.7414890 (accessed September 20, 2017)

[10] Reshetnikova, G. N., Khabibulina, N. Yu., Kotcubinskiy, V. P., Polonskaya, M. S. Synthesis of control actions with aggregate model. Proc. of 2016 International Siberian Conference on Control and Communications (SIBCON-2016). Moscow, IEEE, 2016. 5 p. Available at: http://dx.doi.org/10.1109/SIBCON.2016.7491806 (accessed September 15, 2017)

[11] Reshetnikova, G. N., Khabibulina, N. Yu., Kotcubinskiy, V. P., Polonskaya, M. S. Servo systems with incomplete information. Proc. 2017 International Siberian Conference on Control and Communications (SIBCON-2017). Astana, Kazakhstan, IEEE, 2017, 4 p. Available at: http://ieeexplore.ieee.org/document/7998569/ (accessed September 25, 2017) 